Int. J. Dev. Biol. 53: 1075-1079 (2009)

doi: $10.1387 / \mathrm{ijdb} .072474 \mathrm{ab}$

\title{
Dynamic expression pattern of distinct genes in the presomitic and somitic mesoderm during Xenopus development
}

\author{
AUDREY BOURDELAS\#, HONG-YAN LI\#, CLÉMENCE CARRON and DE-LI SHI* \\ Groupe de Biologie Expérimentale, Laboratoire de Biologie du Développement, CNRS UMR 7622, \\ Université Pierre et Marie Curie, Paris, France
}

\begin{abstract}
Whole-mount and sectioned in situ hybridization was used to identify genes with restricted expression pattern in the presomitic and somitic mesoderm during Xenopus early development. Here we report the dynamic expression pattern of six distinct genes differentially expressed in these regions. These include Xenopus homologues of purine nucleoside phosphorylase, acetylcholine receptor, aspartate aminotransferase, glycine amidinotransferase and brain and muscle isoform creatine kinases. Purine nucleoside phosphorylase was initially expressed in the marginal zone at gastrula stage and then localized to the tail bud. Although the other genes showed no significantly localized expression at gastrula or neurula stages, they are progressively restricted in the somitic mesoderm as development proceeds. A common feature for these genes is that their deficiency in humans leads to an impairment of several tissue or cell functions. An analysis of their expression pattern could provide information regarding their implication in early development.
\end{abstract}

KEY WORDS: purine nucleoside phosphorylase, acetylcholine receptor, aspartate aminotransferase, glycine amidinotransferase, creatine kinase, somite, mesoderm, Xenopus

\section{Introduction}

During the early process of skeletal muscle cell differentiation, the specification and commitment of myogenic cells are initiated by the activation of different members of the bHLH myogenic gene family including Myf5, MyoD, myogenin and Mrf4. These genes exhibit distinct expression pattern and have specific function during the specification and differentiation process of muscle cells (reviewed in Buckingham, 2006). They have the activity to induce myogenic conversion in a variety of cultured cells. During the differentiation process of muscle cells, the expression and activity of other genes are required and mutations of these genes result in dysfunction of muscle cells. Thus, identification and analysis of novel genes expressed in the presomitic and somitic mesoderm can provide insight into the molecular mechanism involved in myogenesis and muscle cell function. We have previously used whole-mount in situhybridization approach to identify genes with restricted expression pattern during early development (Bourdelas et al., 2004). The present study reports the identification and analysis of the spatial and temporal expression pattern of six genes during early development. Two of these genes (purine nucleoside phosphorylase and aspartate aminotransferase) are novel genes in Xenopus. Although the other genes have been previously characterized in Xenopus, there was no detailed analysis of their early expression pattern. The cDNA clones corresponding to these genes were obtained by sequencing a gastrula cDNA library and blast search indicates that full-length cDNA sequences corresponding to these clones could be found in the database. Their expression pattern at different developmental stages was analyzed both by whole-mount and sectioned in situ hybridization.

Abbreviations used in this paper: AGAT, L-arginine:glycine amidinotransferase; bHLH, basic helix-loop-helix; PNP, purine nucleoside phosphorylase;

\footnotetext{
*Address correspondence to: De-Li Shi. Groupe de Biologie Expérimentale, Laboratoire de Biologie du Développement, CNRS UMR 7622, Université Pierre et Marie Curie, 9 quai Saint-Bernard, 75005 Paris, France. Fax: 33-1-4427-3451. e-mail: de-li.shi@upmc.fr
}

Supplementary Material for this paper (six sequence alignments) is available at: http://dx.doi.org/10.1387/ijdb.072474ab

\#Note: Both authors contributed equally to this work.

Accepted: 1 April 2008. Published online: 29 June 2009.

ISSN: Online 1696-3547, Print 0214-6282

(๑) 2009 UBC Press

Printed in Spain 

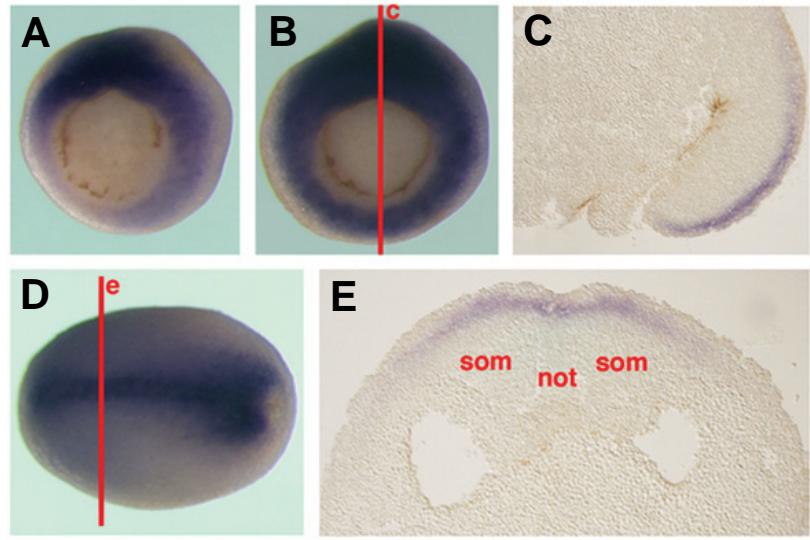

E
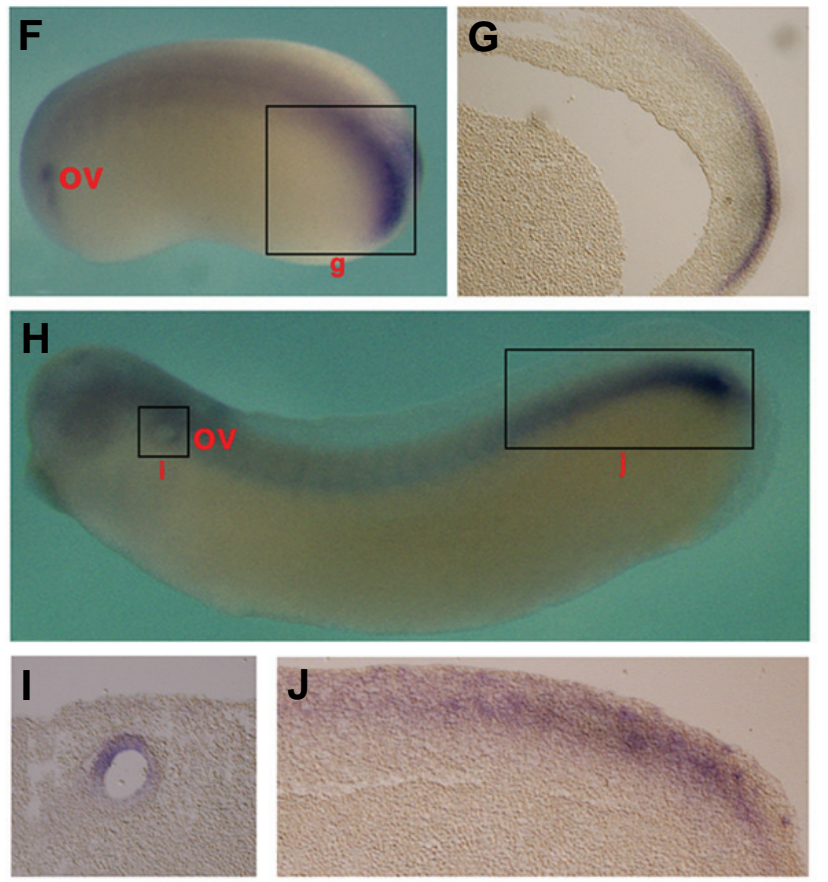

\section{Results and Discussion}

\section{Purine nucleoside phosphorylase}

Purine nucleoside phosphorylase (PNP) catalyzes the phosphorolysis of nucleosides and deoxynucleosides, generating ribose 1-phosphate and the purine base, which is an important step of purine metabolism pathway. The lack of such an activity in humans leads to an impairment of T-cell function (Bzowska et al., 2000). The Xenopus homologue of PNP has not been described previously. Sequence alignment (Supplementary Fig. S1) indicates that the Xenopus protein exhibits $65 \%$ and $68 \%$ overall identity to its mouse and human homologue, respectively. The gene shows dynamic expression pattern during early development. It is first strongly expressed in the dorsal marginal zone and relatively weakly in the lateral and ventral marginal zones at the early gastrula stage (Fig. 1A). As gastrulation proceeds, the strong expression also extends to the lateral and ventral domain (Fig. 1B). In sectioned gastrula, the transcripts were localized to the deep layer of the prospective neural plate (Fig. 1C). This expression persists until at the end of gastrulation (not shown). At
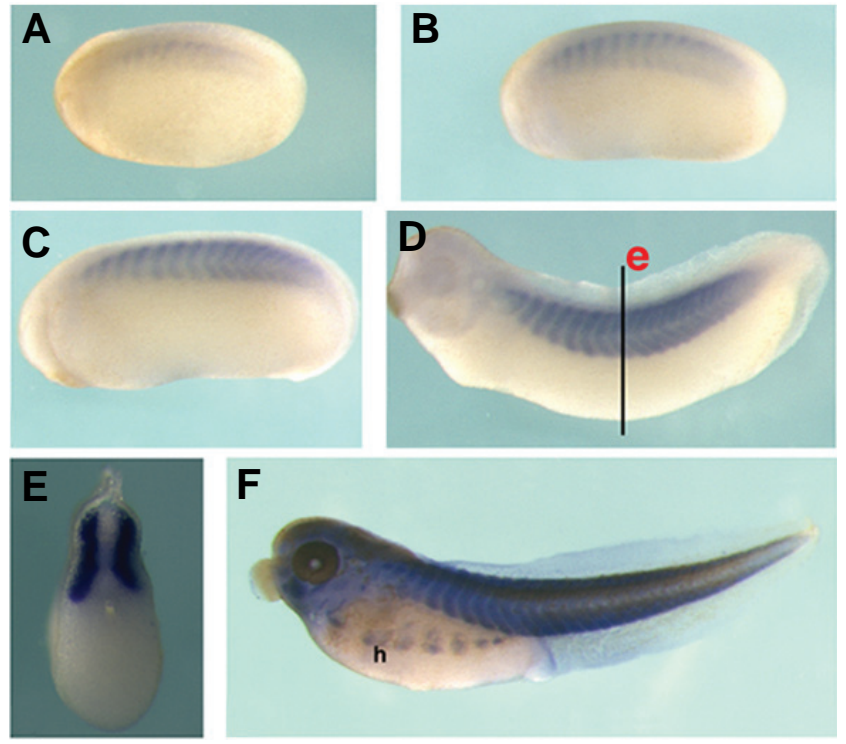

Fig. 1 (Left). Expression of purine nucleoside phosphorylase. (A) Vegetal view of an early gastrula (stage 10.5), with a strong expression in the dorsal marginal zone. (B) Vegetal view of a stage 11 gastrula. The transcripts are detected in the entire marginal zone. (C) Section from the line in (B), showing expression in the deep layer of dorsal marginal zone cells. (D) Dorsal view of a stage 15 early neurula. Expression is localized to the posterior region and the entire neural plate. (E) Section from the line in (D), showing expression in the deep layer of neural plate and absence of expression in the somites and notochord. (F) Lateral view of a stage 22 early tail-bud embryo, with the expression essentially detected in the tail bud and the forming otic vesicle (OV). (G) Section from inset in (F), showing expression in deep cells of the tail bud. (H) Lateral view of a stage 28 tail-bud embryo. The expression is restricted in the posterior tip of the tail bud and in the otic vesicle (OV). (I) Section from inset in (H), with higher magnification of the otic vesicle. (J) Section corresponding to inset in (H), showing expression in the tail bud including both unsegmented posterior mesoderm and neural precursor cells.

Fig. 2 (Right). Expression of acetylcholine receptor $\boldsymbol{\alpha 1 a}$. Lateral view for all embryos, except in (E) which is a section at the level of the line in (D). (A) Stage 20 late neurula with weak expression in the trunk somites. (B) Stage 22. (C) Stage 25. (D,E) Stage 32. (F) A stage 41 larva. h, hypaxial muscle cells.

neurula stage, it is strongly expressed in the posterior region above the blastopore, and in the deep layer of the neural plate, but not in the somites or notochord (Fig. 1D, E). From the end of neurulation or the early tail-bud stage onward, the expression is essentially restricted to the posterior tail-bud region composed of both unsegmented mesoderm and posterior neural tissue, and in the forming otic vesicle (Fig. 1F-J).

\section{Acetylcholine receptor}

The nicotinic acetylcholine receptor is a ligand-gated channel that mediates signalling at the vertebrate neuromuscular junction and is consisted of four subunits. In Xenopus, there are two $\alpha$ subunits, $\alpha 1 \mathrm{a}$ and $\alpha 1 \mathrm{~b}$, encoded by distinct genes (Hartman and Claudio, 1990). Although these two $\alpha$ subunits were found to be muscle-specific, there is no detailed description of their temporal and spatial expression during early development (Baldwin et al., 1988; Hartman and Claudio, 1990). The cDNA clone 6D08 that we 
identified corresponds to $\alpha 1 \mathrm{a}$. Sequence alignment indicates that it is highly conserved in vertebrates (Supplementary Fig. S2). It is a maternal mRNA without any detectable localized expression during gastrulation and neurulation (not shown). At the end of neurula stage, it is weakly detected in the formed somites, but not in the posterior presomitic mesoderm (Fig. 2A). This suggests that acetylcholine receptor may function as early as the first somite forms. The somitic expression persists and becomes strong as development proceeds (Fig. 2B-D). In sectioned embryo at the trunk region, it is clear that $\alpha 1$ a subunit is somite-specific and is localized in all the compartments of the somite (Fig. 2E). At larval stage, the expression is also detected in the hypaxial muscle cells and head muscle cells (Fig. 2F).

\section{Aspartate aminotransferase}

The cDNA clone 27F11 encodes a protein corresponding to Xenopus aspartate aminotransferase. This protein is highly conserved among vertebrates and the Xenopus protein shows $81 \%$ and $80 \%$ overall identity to its mouse and human homologue, respectively (Supplementary Fig. S3). This enzyme is involved in liver glyceroneogenesis (Ferris and Williamson, 1978), but its expression pattern and function during early development in different species remains unknown. At gastrula and neurula stages, there is no localized expression (not shown), but restricted expression in the formed somites becomes evident at the end of neurulation or early tail-bud stage (Fig. 3A). As development proceeds, strong expression can be found in the somites, but not in the posterior presomitic mesoderm. Beginning at stage
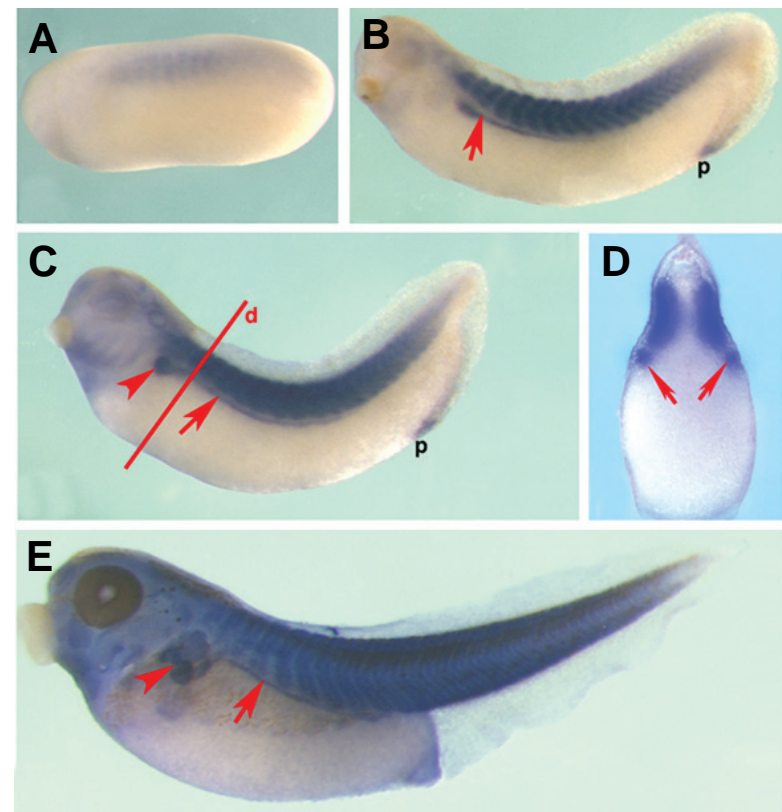

30 , it can be also detected in the proctodium and pronephric duct, but not yet in the pronephros anlag (Fig. 3B). This expression pattern then persists during late development, with an additional expression site in the pronephros analg from stage 32 (Fig. 3C, D) onward to at least larval stage (Fig. 3E). At these stages, the transcripts could be also detected diffusely in the ventral region of the branchial arches and in the heart primodium (Fig. 3C, E).

\section{Glycine amidinotransferase}

Glycine amidinotransferase or L-arginine:glycine amidinotransferase (AGAT) is one of the proteins strongly conserved (Supplementary Fig. S4) and is involved in the synthesis and uptake of creatine and thus in energy metabolism. This gene exhibits dynamic expression pattern during development. The first detectable expression of $A G A T$ is localized in the marginal zone at the early gastrula stage, with a higher level in the dorsal region (Fig. 4A). At the end of gastrulation, it is weakly expressed in the dorsal region corresponding to the presumptive neural plate (Fig. 4B). At the end of neurulation, it is diffusely expressed in the somitic mesoderm and in the ventral endoderm at the trunk region (Fig. 4C). This expression pattern persists during later development and extends to the entire formed somites and the endodermal mass (Fig. 4D, E). In sectioned embryo at the trunk region, it is clear that $A G A T$ is localized in the somite and endodermal mass, as well as weakly in the notochord, but not in the neural tube and lateral plate mesoderm (Fig. 4F). At larval stage, strong expression remains in the somites, while the endodermal expression becomes relatively weak. A weak expression can be also found in the hypaxial muscle cells (Fig. 4G). The cDNA clone 4A07 obtained in this study was identical to a previously identified sequence. However, some differences exist between previous
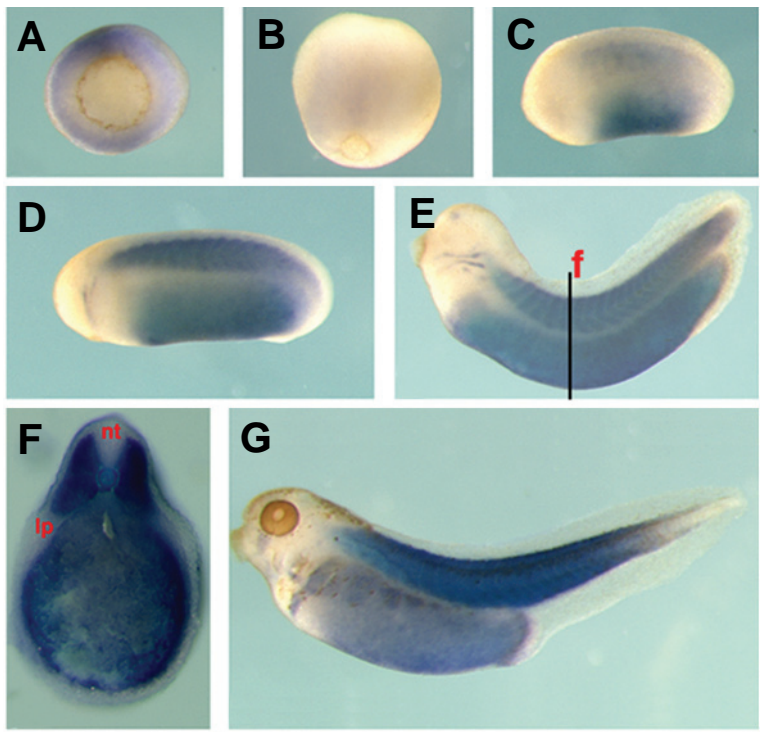

Fig. 3 (Left). Expression pattern of aspartate aminotransferase. Lateral view for all embryos, except for (D). (A) A stage 23 early tail-bud embryo. (B) Stage 30, with strong expression in the somites, pronephric duct (arrow). Expression also appears in the proctodium (p). (C) Stage 35. Additional expression site is found in the pronephros anlag (arrow head). (D) Section from the line in (C), showing expression in somites and pronephros (arrows). (E) Stage 41

Fig. 4 (Right). Expression of glycine amidinotransferase. (A) Vegetal view of a stage 10.5 early gastrula showing the expression in the marginal zone. (B) Dorsal view of a stage 12 late gastrula with weak expression in the neural plate. (C) Stage 22, lateral view. Expression can be seen in the somites and endoderm. (D) Lateral view of a stage 25 tail-bud embryo. (E) Stage 35, lateral view. (F) A section made at the level of the line in (E). (G) A stage 41 larval stage embryo. nt, neural tube; Ip, lateral plate. 

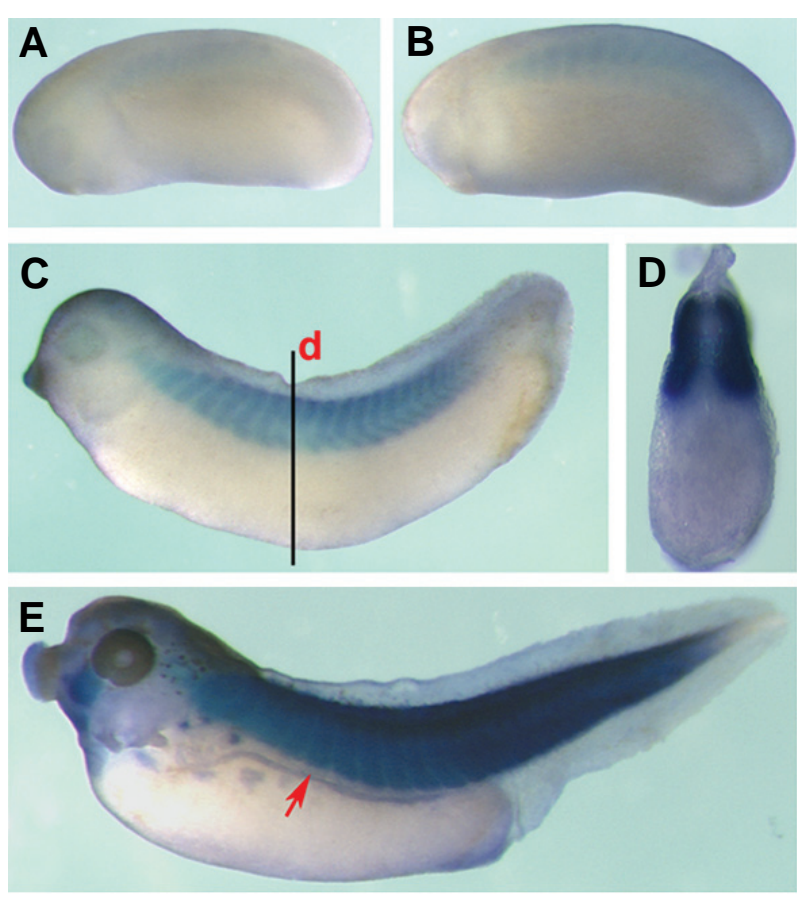
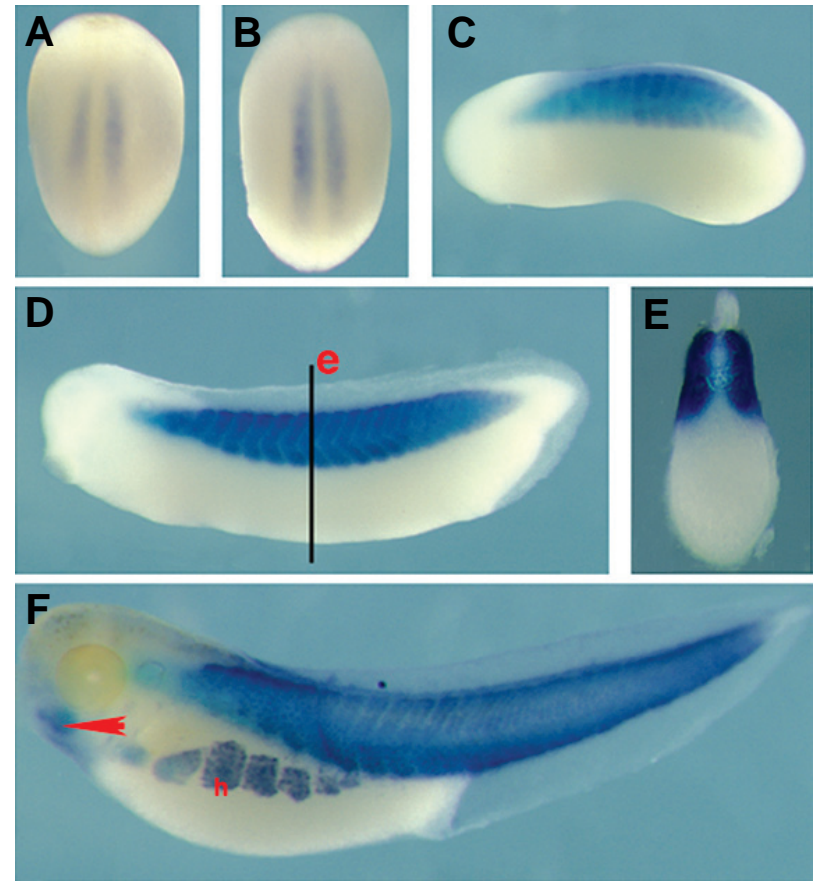

Fig. 5 (Left). Expression pattern of the brain isoform creatine kinase. (A) Lateral view of a stage 23 tail-bud embryo. (B) Stage 25, lateral view. (C) Stage 30, lateral view. (D) Section at the level of the line in (C). (E) A stage 41 larva, lateral view. Arrow indicates the pronephric duct.

Fig. 6 (Right). Expression pattern of the muscle isoform creatine kinase. (A) Stage 18, dorsal view. (B) Stage 20, dorsal view. (C) Stage 25, lateral view. (D) Stage 30, lateral view. (E) Section at the level of the line in (D). (F) A stage 41 larva, lateral view. h, hypaxial muscle cells. Arrow indicates head muscle cells.

and our present study. Previous analysis by whole-mount in situ hybridization detected Xenopus AGAT expression in the notochord and gut endoderm, but not in the somites (Zhao et al., 2001). We cannot explain this discrepancy, one possibility might be the probes used in different study. In the present study, we have used the full-length cDNA sequence as a template for probe synthesis (Table 1; Supplementary Fig. S4).

\section{Creatine kinase}

As AGAT, creatine kinase (CK) enzymes are remarkably conserved (Supplementary Fig. S5 and S6) and are also involved in energy metabolism. They catalyse the reversible phosphorylation of creatine from ATP. In higher vertebrates, there are two subunits, the brain and muscle isoforms, encoded by two distinct genes (Robert et al., 1990). In Xenopus, immunochemical characterization of these two isoforms has been described for larval

TABLE 1

\section{CDNA CLONES IDENTIFIED BY WHOLE-MOUNT IN SITU HYBRIDIZATION SCREENING}

\begin{tabular}{llll} 
cDNA clones & Genes & Accession numbers & Probe regions \\
\hline $21 \mathrm{A04}$ & Purine nucleoside phosphorylase & $\mathrm{BC} 054317$ & 3'-UTR \\
6D08 & Acetylcholine receptor $\alpha 1 a$ & $\mathrm{X} 07067, \mathrm{X} 17244$ & aa 102 onward \\
27F11 & Aspartate aminotransferase & $\mathrm{BC} 106292$ & aa 331 onward \\
4A07 & Glycine amidinotransferase & $\mathrm{AF} 187863, \mathrm{BC} 047973$ & aa 24 onward \\
$6 \mathrm{~F} 01$ & Creatine kinase, brain isoform & $\mathrm{BC} 042282$ & aa 268 onward \\
& Creatine kinase, muscle isoform & $\mathrm{BC042249}$ & aa 94 to 342 \\
\hline
\end{tabular}

and adult tissues (Robert et al., 1990; 1991a, b). However, mRNA expression data of these two genes during early development has been lacking. The cDNA clone 6F01 showed an identical sequence with the Xenopus brain isoform creatine kinase found in the database (Table 1). The predicted amino acid sequence shows $85 \%$ overall identity with the Xenopus muscle isoform creatine kinase. To better distinguish the expression pattern between these two isoforms, we amplified by PCR a cDNA fragment corresponding to the muscle isoform and used it as a probe. The results indicate that they exhibit both overlapping and distinct expression. The brain isoform is uniformly expressed in the ectoderm during gastrulation and neurulation (not shown). Beginning from the tail-bud stage (stage 25), it can be weakly detected in the somites (Fig. 5A, B). As development proceeds, strong expression is detected in the somites (Fig. 5C, D). At larval stage, strong expression persists in the somites, other sites such as head muscle, the heart region and the pronephric duct also express the transcripts corresponding to the brain isoform (Fig. $5 \mathrm{E})$.

The muscle isoform creatine kinase is restricted in the somites. It is not detectable during gastrulation (not shown). At neurula stage, it is expressed in the formed somites at the trunk region (Fig. 6A, B). Strong expression in the somites persists as development proceeds (Fig. 6C-F). At larval stage, it is also strongly expressed in the head muscle and in the hypaxial muscle cells migrating away from the somites, a diffuse expression could be observed in the heart region (Fig. 6G). Unlike the brain isoform, the muscle isoform is not expressed in the pronephros. Thus, this expression pattern is much similar or identical as other muscle 
marker genes such as muscle actin and myosine light chain. These results suggest that the brain isoform creatine kinase is also strongly expressed in the somites while the muscle isoform exhibits indeed a restricted expression in the somites. Although the two isoforms have a higher overall identity at the protein level, the nucleotide sequences of the coding region are significantly divergent, and the 3' non-coding regions do not have any similarity. Thus, we can exclude the possibility of a cross-reactivity.

\section{Materials and Methods}

Whole-mount in situhybridization screening of a gastrula cDNA library was previously described (Bourdelas et al., 2004). The muscle isoform creatine kinase was PCR amplified according to published sequence (accession number BC042249) using the following primers: upstream, (5'-ATTGAGGATCGTCACGGTGG-3'; downstream, 5'GCGTCTGAAGACTTCTTTCAT-3'. The PCR product was cloned in the T-easy vector, linearized by $\mathrm{NCO}$ I and transcribed with SP6 RNA polymerase to obtain antisense probe.

\section{Acknowledgements}

This work was supported by CNRS, University Pierre and Marie Curie and by a grant from The Association Française contre les Myopathies to D.L. Shi. H. Y. Li was supported by a post-doctoral fellowship from CNRS.

\section{References}

BALDWIN, T.J., YOSHIHARA, C.M., BLACKMER, K., KINTNER, C.R. and BUR-
DEN, S.J. (1988). Regulation of acetylcholine receptor transcript expression during development in Xenopus. J. Cell Biol. 106: 469-478.

BOURDELAS, A., LI, H.Y., BOUCAUT, J.C. and SHI, D.L. (2004). Identification of distinct genes with restricted expression in the somitic mesoderm in Xenopus embryo. Gene Expr. Patterns 4: 695-699.

BUCKINGHAM, M. (2006). Myogenic progenitor cells and skeletal myogenesis in vertebrates. Curr. Opin. Genet. Dev. 16: 525-532.

BZOWSKA, A., KULIKOWSKA, E. and SHUGAR, D. (2000). Purine nucleoside phosphorylases: properties, functions, and clinical aspects. Pharmacol. Ther. 88: 349-425.

FERRIS, P. and WILLIAMSON, D.H. (1978). Evidence for the participation of aspartate aminotransferase in hepatic glucose synthesis in the suckling newborn rat. Biochem. J. 176: 335-338.

HARTMAN, D.S. and CLAUDIO, T. (1990). Coexpression of two distinct muscle acetylcholine receptor a-subunits during development. Nature 343: 372-375.

ROBERT, J., WOLFF, J., JUAKLI, H., GRAF, J.D., KARCH, F. and KOBEL, H.R. (1990). Developmental expression of the creatine kinase isozyme system of Xenopus: maternally derived CK-IV isoform persists far beyond the degradation of its maternal mRNA and into the zygotic expression period. Development 108: 507-514.

ROBERT, J., BARANDUN, B. and KOBEL, H.R. (1991a). A Xenopus laevis creatine kinase isozyme (CK-III/III) expressed preferentially in larval striated muscle: cDNA sequence, developmental expression and subcellular immunolocalization. Genet. Res. 58: 35-40.

ROBERT, J., DU PASQUIER, L. and KOBEL, H.R. (1991b). Differential expression of creatine kinase isozymes during development of Xenopus laevis:an unusual heterodimeric isozyme appears at metamorphosis. Differentiation 46: 23-34.

ZHAO, H., CAO, Y. and GRUNZ, H. (2001). Expression of Xenopus L-arginine: glycine amidinotransferase (XAT) during early embryonic development. Dev. Genes Evol. 211: 358-360.

\section{For all the latest on Pattern Formation research, see our latest Special Issue edited by C.-M. Chuong and M.K. Richardson.}

5 yr ISI Impact Factor $(2008)=3.271$

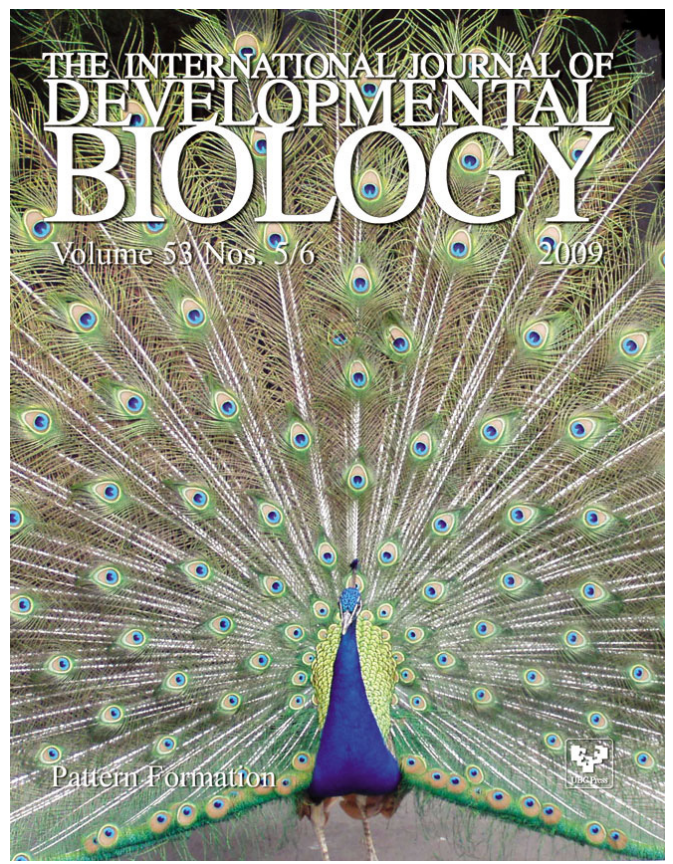




\section{Further Related Reading, published previously in the Int. J. Dev. Biol.}

See our Special Issue Invasion, edited by Marc Mareel and Juan Arechaga at: http://www.ijdb.ehu.es/web/contents. php?vol=48\&issue =5-6

A comparative analysis of Meox1 and Meox2 in the developing somites and limbs of the chick embryo

Susan Reijntjes, Sigmar Stricker and Baljinder S. Mankoo

Int. J. Dev. Biol. (2007) 51: 753-759

Myoskeletin, a factor related to Myocardin, is expressed in somites and required for hypaxial muscle formation in Xenopus

Hui Zhao, Martha L. Rebbert and Igor B. Dawid

Int. J. Dev. Biol. (2007) 51: 315-320

Spatiotemporal expression of the creatine metabolism related genes agat, gamt and ct1 during zebrafish embryogenesis

Lifeng Wang, Ying Zhang, Ming Shao and Hongwei Zhang

Int. J. Dev. Biol. (2007) 51: 247-253

Myoskeletin, a factor related to Myocardin, is expressed in somites and required for hypaxial muscle formation in Xenopus

Hui Zhao, Martha L. Rebbert and Igor B. Dawid

Int. J. Dev. Biol. (2007) 51: 315-320

Xtbx6r, a novel T-box gene expressed in the paraxial mesoderm, has anterior neural-inducing activity

Shigeharu Yabe, Shunsuke Tazumi, Jun Yokoyama and Hideho Uchiyama

Int. J. Dev. Biol. (2006) 50: 681-689

Bowline, a novel protein localized to the presomitic mesoderm, interacts with Groucho/TLE in Xenopus

Akiko Kondow, Keisuke Hitachi, Tempei Ikegame and Makoto Asashima

Int. J. Dev. Biol. (2006) 50: 473-479

Bowline, a novel protein localized to the presomitic mesoderm, interacts with Groucho/TLE in Xenopus

Akiko Kondow, Keisuke Hitachi, Tempei Ikegame and Makoto Asashima

Int. J. Dev. Biol. (2006) 50: 473-479

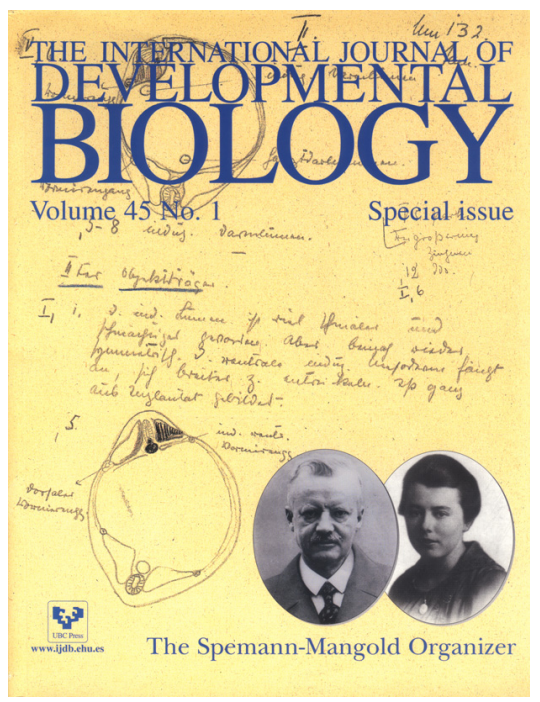

5 yr ISI Impact Factor $(2008)=3.271$

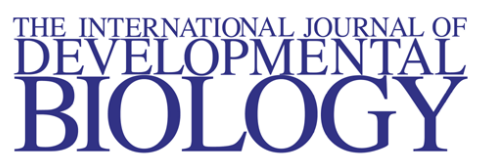

Volume 48 Nos. $5 / 6$

Special issue

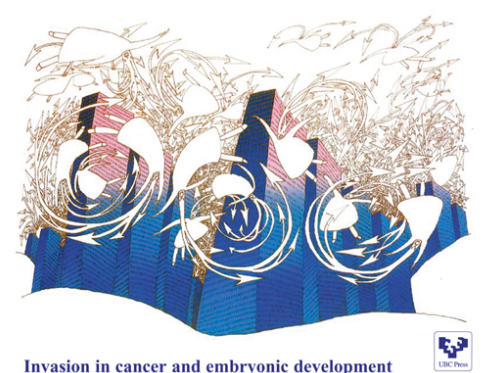

